\title{
COUP-TFII orchestrates venous and lymphatic endothelial identity by homo- or hetero-dimerisation with PROX1
}

\author{
Xabier L. Aranguren ${ }^{1}$, Manu Beerens ${ }^{1}$, Giulia Coppiello ${ }^{1}$, Cornelia Wiese ${ }^{2}$, Ine Vandersmissen ${ }^{1}$, \\ Antonio Lo Nigro ${ }^{3}$, Catherine M. Verfaillie ${ }^{3}$, Manfred Gessler ${ }^{2}$ and Aernout Luttun ${ }^{1, *}$ \\ ${ }^{1}$ Department of Cardiovascular Sciences, Molecular and Vascular Biology Research Unit, Endothelial Cell Biology Unit, KU Leuven, B-3000 Leuven, \\ Belgium \\ ${ }^{2}$ Theodor-Boveri-Institut, Biocenter, Developmental Biochemistry, Würzburg University, D-97074 Würzburg, Germany \\ ${ }^{3}$ Stem Cell Institute Leuven, KU Leuven, B-3000 Leuven, Belgium \\ *Author for correspondence (aernout.luttun@med.kuleuven.be)
}

Accepted 14 December 2012

Journal of Cell Science 126, 1164-1175

(๑) 2013. Published by The Company of Biologists Ltd

doi: 10.1242/jics. 116293

\section{Summary}

Endothelial cell (EC) identity is in part genetically predetermined. Transcription factor NR2F2 (also known as chicken ovalbumin upstream promoter transcription factor II, COUP-TFII) plays a key role in EC fate decision making; however, many of the underlying mechanisms remain enigmatic. In the present study, we demonstrate that NR2F2 differentially regulates gene expression of venous versus lymphatic ECs (LECs) and document a novel paradigm whereby NR2F2 homodimers induce a venous EC fate, while heterodimers with the LEC-specific transcription factor PROX1 instruct LEC lineage specification. NR2F2 homodimers inhibit arterial differentiation in venous ECs through direct binding to the promoter regions of the Notch target genes HEY1 and HEY2 (HEY1/2), whereas NR2F2/PROX1 heterodimers lack this inhibitory effect, resulting at least in part in non-canonical HEY1/2 expression in LECs. Furthermore, NR2F2/PROX1 heterodimers actively induce or are permissive for the expression of a major subset of LEC-specific genes. In addition to NR2F2/PROX1 heterodimerisation, the expression of HEY1 and some of these LEC-specific genes is dependent on PROX1 DNA binding. Thus, NR2F2 homodimers in venous ECs and NR2F2/PROX1 heterodimers in LECs differentially regulate EC subtype-specific genes and pathways, most prominently the Notch target genes HEY1/2. This novel mechanistic insight could pave the way for new therapeutic interventions for vascular-bed-specific disorders.

Key words: COUP-TFII, Prox1, Endothelial specification, Notch signalling

\section{Introduction}

ECs lining the inside of veins, arteries and lymphatic vessels feature remarkable functional, molecular and morphological heterogeneity. While these characteristics are partly acquired by exposure to environmental cues, genetic studies support the concept that EC identity is also intrinsically predetermined (Aird, 2007a; Aird, 2007b). Arterial ECs (AECs) express EphrinB2, while its receptor EphB4 is present on their venous counterparts (VECs), a polarity that is established before the onset of blood flow (Wang et al., 1998). Similarly, LECs uniquely express the transcription factor Prox1, before the lymphatic system becomes functional (Srinivasan et al., 2007). Many other molecular players participate in EC fate decisions, including Notch, sonic hedgehog (Shh), vascular endothelial growth factor (VEGF), angiopoietins, transforming growth factor- $\beta$, Wnt, Sry-related HMG box (Sox), forkhead box (Fox)C1/2, adrenomedullin, Spreds and phosphatidylinositol-3 kinase (Corada et al., 2010; Oliver and Srinivasan, 2010; Swift and Weinstein, 2009). Often they are organised hierarchically, such as the Shh-VEGF-Notch cascade establishing an arterial phenotype.

The formidable challenge of unravelling the molecular complexity of AEC, VEC and LEC differentiation is further enhanced by the notion that several factors are commonly expressed amongst EC subtypes. Notch receptors and EphrinB2, thought to be restricted to AECs, were later found on LECs (Geudens et al., 2010; Kang et al., 2010; Shawber and Kitajewski, 2008). Since LECs originate by transdifferentiation from VECs, it is not surprising that they also share some molecules (Srinivasan et al., 2007; Srinivasan et al., 2010). One of these is the transcription factor COUP-TFII, which we will refer to as 'NR2F2'. It belongs to a family of nuclear orphan receptors that play key roles in neuronal development, organogenesis, cell fate determination and metabolic homeostasis ( $\mathrm{Li}$ et al., 2009; Tsai and Tsai, 1997). Targeted NR2F2 deletion in mice revealed its critical role in cardiovascular development (Pereira et al., 1999). Furthermore, EC-specific deletion demonstrated a cellautonomous function of NR2F2 in establishing VEC identity during development (You et al., 2005). Recently, NR2F2 was shown to be required for induction of Prox 1 - a master-switch for LEC differentiation - and thus for LEC transdifferentiation from VECs (Srinivasan et al., 2007; Srinivasan et al., 2010).

An intriguing question is how a transcription factor, commonly expressed by distinct EC subtypes, can regulate distinct EC fates and which pathways lay downstream of NR2F2 in the two EC subtypes. Based on the observation that NR2F2-deficient VECs featured ectopic expression of neuropilin (Nrp)1 - a VEGF co-receptor - in 
addition to Notch1 and its downstream target Hey1 in mice, it was proposed that NR2F2 imposes a VEC phenotype by blocking activation of the arterial VEGF-Notch cascade (You et al., 2005). Another model suggested that NR2F2 cooperates with PROX1 in LECs to interrupt the VEGF-Notch pathway to prevent AEC specification and induce a LEC fate (Kang et al., 2010). However, some important mechanistic riddles remain unresolved. While both models agree on NR2F2's inhibitory role on the arterialising VEGFNotch pathway, the underlying mechanisms of Notch inhibition are not fully established in VECs and LECs (Kang et al., 2010; Srinivasan et al., 2010; Swift and Weinstein, 2009). While the expression of Notch pathway members was documented in LECs (Kang et al., 2010; Shawber et al., 2007; Shawber and Kitajewski, 2008; Zheng et al., 2011), it remains unclear which mechanisms drive this expression and whether or how they play a role in LEC specification, maintenance and function. Conflicting results have been reported, some suggesting a positive role for Notch signalling in lymphangiogenesis during zebrafish development (Geudens et al., 2010) and in neonatal mice (Niessen et al., 2011), others demonstrating an inhibitory role in adult mice (Zheng et al., 2011). While these discrepancies could be explained by age- and/or species-dependent differences, another complication is that Notch signalling occurs through different mechanisms. Notch signalling can proceed via the canonical pathway - involving nuclear translocation of the cleaved Notch receptor and binding to RВРЈк - or independent of it (Sanalkumar et al., 2010). The (relative) importance of both pathways in LECs, however, remains a matter of debate (Geudens et al., 2010; Srinivasan et al., 2010).

One potential mechanism by which a common transcription factor may acquire EC subtype-specific regulatory properties is by its (physical) association with another transcription factor that is uniquely expressed in one of the EC subtypes. Recently, two labs independently demonstrated that NR2F2 forms functional heterodimers with the LEC-restricted transcription factor PROX1 in LECs (Lee et al., 2009; Yamazaki et al., 2009). Furthermore, both transcription factors were shown to drive the expression of a LEC gene subset in a cooperative manner, while other genes were regulated independently by either factor alone (Lee et al., 2009). However, these studies did not address whether heterodimerisation with PROX1 in LECs was responsible for a differential effect of NR2F2 on gene expression in LECs versus VECs.

Here, using a combination of loss- and gain-of-function studies in human ECs, we unravel a novel mechanism whereby NR2F2 differentially regulates transcription of Notch target genes $H E Y 1$ and HEY2 (HEY1/2), in VECs and LECs by directly acting on their promoters. This interaction resulted in a transcriptional block on $H E Y$ expression in VECs, but not in LECs. We further show that NR2F2 switched from forming homodimers in VECs to heterodimerisation with PROX1 in LECs, the latter resulting in the release of the transcriptional block on $H E Y$ expression. HEY expression in LECs was at least partly independent of canonical Notch signalling. Moreover, the homo-heterodimerisation switch was responsible for the differential regulation by NR2F2 of a major subset of LEC and VEC genes.

\section{Results}

NR2F2 directs distinct transcriptional/functional programs in VECs and LECs

As shown here (Fig. 1A,B) and previously by others, while PROX1 expression is restricted to LECs, NR2F2 is commonly expressed in VECs (You et al., 2005) and LECs during development (Lin et al., 2010; Srinivasan et al., 2010) and adulthood (Lee et al., 2009; Lin et al., 2010; Yamazaki et al., 2009), raising the question how one transcription factor can regulate and/or maintain both EC identities. To globally understand differential gene regulation, we performed a genome-wide comparative transcriptome analysis of human umbilical vein ECs (HUVECs) and a homogenous LEC population in the presence or absence of NR2F2. Despite a similar knockdown efficiency ( $\sim 80 \%$ at RNA level) in both EC types, NR2F2 knockdown in HUVECs and LECs revealed remarkably different changes in their gene expression profile. Up to 204 genes were exclusively regulated by NR2F2 knockdown in HUVECs (e.g. $H E Y 1 / 2), 191$ were exclusively regulated in LECs (e.g. LYVE1, ITG9A), while only 54 genes were commonly regulated in both cell types (e.g. NRP2; Fig. 1C; a complete list of genes significantly affected by NR2F2 knockdown is provided in supplementary material Table S1). Ingenuity Pathway Analysis revealed that genes that were differentially affected by NR2F2 knockdown belonged to distinct pathways (Fig. 1C). Functional annotation analysis identified molecular transport as the most abundant function regulated by NR2F2 in HUVECs, whereas cellular movement was the primary function in LECs (Fig. 1D). In accordance with published data (Lin et al., 2010), cultured LECs migrated less in the absence of NR2F2 in response to VEGF-C (Fig. 1D). Thus, NR2F2 orchestrates distinct transcriptional and functional programs in VECs and LECs.

\section{NR2F2 differently affects $H E Y$ expression in VECs and LECs}

The principal pathway differentially affected by NR2F2 knockdown was the Notch pathway. In endothelial-specific NR2F2-deficient mice, Notch pathway members become ectopically expressed in VECs. This generated a working model whereby NR2F2 regulates VEC identity by blocking the arterial VEGF-Notch cascade; however, how NR2F2 blocks Notch signalling in VECs was not directly tested (You et al., 2005). Consistent with a repressive effect of NR2F2 on Notch signalling, NR2F2 knockdown in HUVECs resulted in a significant upregulation of $\mathrm{NOTCH} 4$, delta-like (DLL)4, HEYI and HEY2 (Fig. 1E). This was accompanied by an arterio-venous expression shift with increased expression of arterial transcript $E F N B 2$ and lowered expression of venous transcripts, EPHB4 and NRP2 (Fig. 1E). Remarkably, in LECs, none of the Notch pathway members was affected by NR2F2 silencing, nor was the expression of EFNB2 (Fig. 1F). Thus, while NR2F2 inhibited expression of Notch pathway members in HUVECs, this did not occur in LECs resulting in a significantly higher expression of Notch targets HEY1/2 in untreated LECs versus HUVECs (Fig. 1G-K).

\section{NR2F2 binding to HEY promoters blocks their expression in HUVECs but not in LECs}

We next assessed how differential gene regulation by NR2F2 in EC subtypes was accomplished, first focusing on the Notch pathway. Since our data suggested a direct repressive effect on HEY1/2 expression in HUVECs, we investigated whether NR2F2 could bind to their promoters to block $H E Y 1 / 2$ transcription. For both promoters we identified four regions each containing an evolutionary conserved putative NR2F2 binding element (Fig. 2A,B). Chromatin immunoprecipitation revealed a true NR2F2 binding site in the proximity of the transcriptional start 

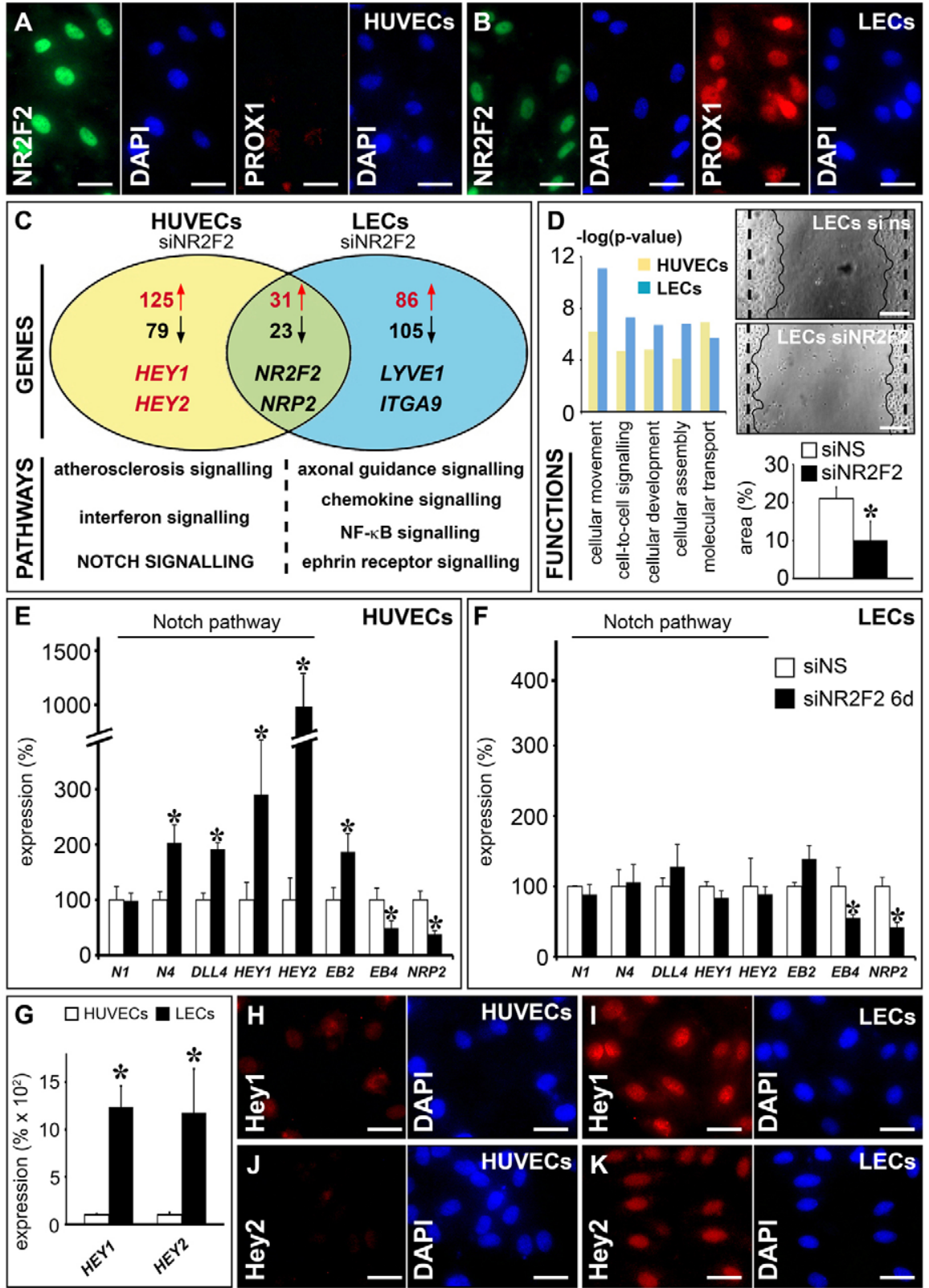

Fig. 1. NR2F2 directs distinct transcriptional/ functional programs in VECs and LECs. $(\mathbf{A}, \mathbf{B})$ Immunofluorescence staining for NR2F2 (green) or PROX1 (red; corresponding DAPI pictures are shown on the right) in HUVECs (A) or LECs (B). (C) Diagram showing the amount of genes and some examples uniquely upregulated or downregulated by NR2F2 siRNA knockdown in HUVECs (yellow) or LECs (blue) or commonly regulated in both cell types (green). Differentially affected pathways are listed below. (D) Diagram showing differential functional regulation by NR2F2 in HUVECs (yellow) and LECs (blue). Insets show impaired VEGF-C induced migration of LECs upon NR2F2 siRNA knockdown (bottom) compared with control (top) in a scratch wound assay. For clarity, dashed lines indicate the original scratch border and wavy lines indicate the moving cell front. The bar diagram represents the mean percentage area \pm s.e.m. occupied by migrated LECs treated with non-silencing (siNS; white) or NR2F2 siRNA (black; $N=3$ independent experiments; ${ }^{*} P<0.05$ versus siNS). (E,F) qRT-PCR on HUVECs (E) and LECs (F) after 6 days (d) of treatment with siNS (white) or NR2F2 siRNA (black), revealing significant upregulation of Notch pathway members NOTCH (N)4, DLL4, HEY1, $H E Y 2$ and arterial marker EFNB2 (EB2) in HUVECs upon NR2F2 knockdown, but no changes in LECs, except for $E P H B 4(E B 4)$ and $N R P 2$, which were downregulated in both cell types. Data are expressed as mean percentage \pm s.e.m. versus control siRNA. $(N=3-4$ independent experiments; $* P<0.05$ versus siNS.) (G) qRT-PCR diagram revealing significantly higher expression of $H E Y 1 / 2$ in LECs (black) versus HUVECs (white). Data are expressed as mean percentage \pm s.e.m. versus HUVECs $(N=3$ independent experiments; ${ }^{*} P<0.05$ versus HUVECs). (H-K) Immunofluorescence staining for HEY1 and HEY2 (red; corresponding DAPI pictures are shown on the right) on cultured HUVECs (H,J) or LECs $(\mathrm{I}, \mathrm{K})$. Scale bars: $20 \mu \mathrm{m}$ in A,B,H-K. site of both genes (corresponding to a direct repeat (DR)4 element in intron 1 of the human HEY1 promoter and an inverted repeat (IR) 1 element upstream of the $5^{\prime}$ untranslated region (UTR) in the human HEY2 promoter; Fig. 2C,D) in HUVECs. Mutation of these sites (to prevent NR2F2 DNA binding) resulted in increased $H E Y 1 / 2$ promoter activity (Fig. 2E), proving that NR2F2 blocks Notch signalling in HUVECs at least partly by direct interaction with these $H E Y 1 / 2$ promoter elements causing transcriptional repression. However, NR2F2 bound the same sites of both genes in LECs (Fig. 2C,D), suggesting that the observed lack of inhibition of Notch targets in LECs was not due to NR2F2's inability to bind the corresponding NR2F2 sites. Of note, NR2F2 binding to the HEY2 promoter was less prominent in LECs (Fig. 2D). Hence, reduced binding to the HEY2 promoter could partly account for the lack of $H E Y 2$ repression in LECs. Thus, while NR2F2 inhibits arterial gene expression in veins through binding to the promoters of $H E Y 1 / 2$ causing their repression, such interactions in LECs are less efficient and/or do not induce transcriptional repression.

\section{HEY expression in LECs is at least in part independent of canonical Notch signalling}

Since the role of canonical Notch signalling in LECs has been debated (Geudens et al., 2010; Srinivasan et al., 2010), we wondered whether basal Notch target gene expression was either the result of canonical regulation (involving generation of Notch intracellular domain or NICD by Notch receptor cleavage and subsequent association with $\mathrm{RBPJ \kappa}$ to form a transcriptional regulatory complex), or rather $\mathrm{NICD/RBPJ \kappa -independent.} \mathrm{In}$ support of the latter hypothesis, RBPJא siRNA knockdown or treatment with DAPT $\{\mathrm{N}-[\mathrm{N}-(3,5-$ difluorophenacetyl)-L-alanyl]S-phenylglycine t-butyl ester; a Notch receptor cleavage inhibitor\} did not change HEY1/2 expression in LECs (Fig. 2F,G). Thus, NICD/RBPJK-independent regulation is 

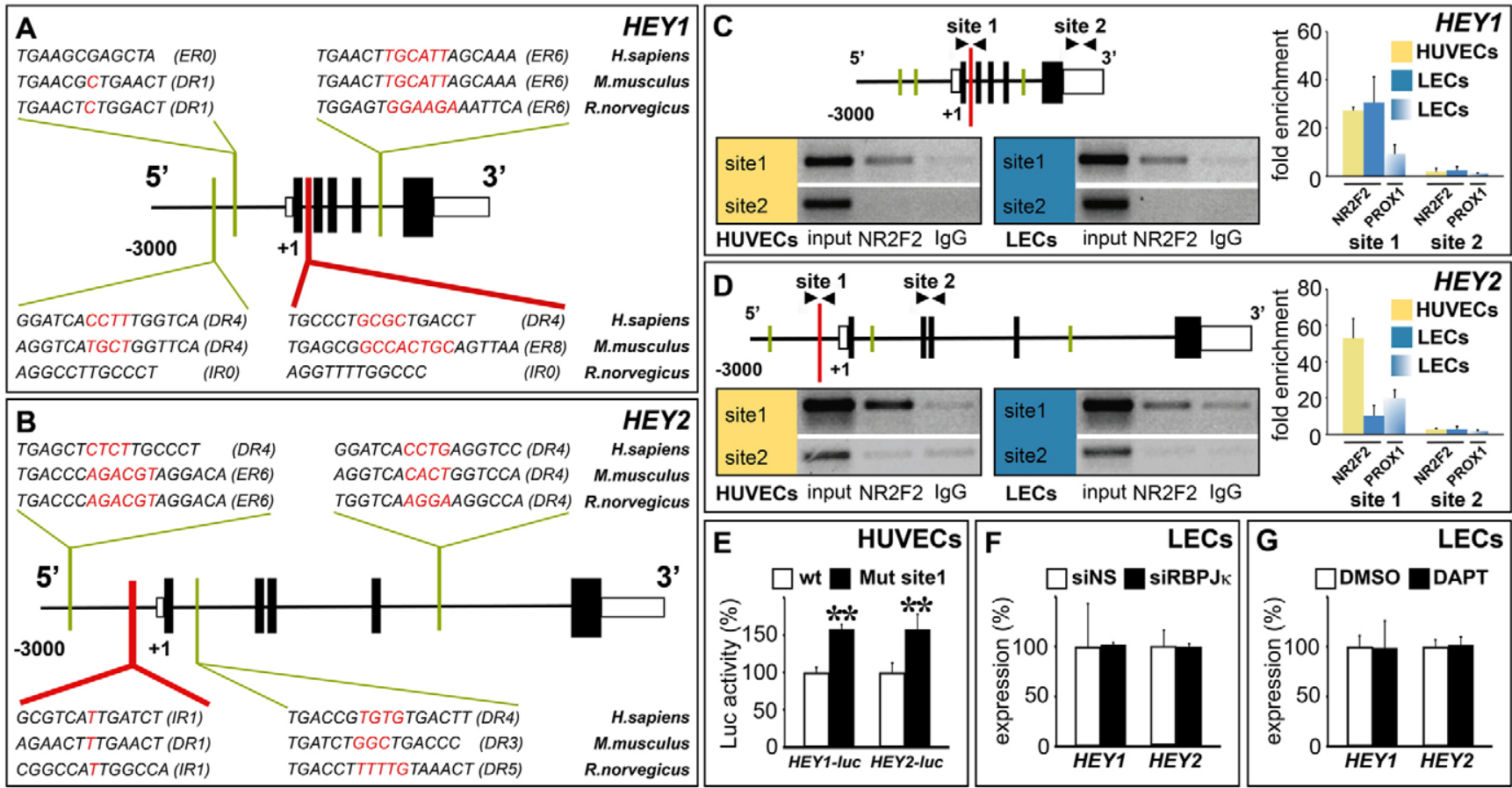

Fig. 2. NR2F2 interacts with HEY promoters in VECs and LECs, and HEY expression in LECs is at least in part independent of canonical Notch signalling. (A,B) Schematic scaled representation of the HEY1 (A) and HEY2 (B) loci highlighting putative NR2F2 binding elements identified in silico by NHRscan and alibaba2.1 software using a combined probability of entering match states of 0.01 to 0.02 . Exons are shown as black boxes, untranslated regions as white boxes and the transcriptional start site as +1 . DR: direct repeat; IR: inverted repeat; ER: everted repeat. Each NR2F2 binding element consists of two halfsites and a variable number of bases in between (which is indicated by the number after DR, IR or ER; lettering in red). Only those putative NR2F2 elements are displayed for which there was an element in the same region (+/- 300-500 bases) in each of the three species (Homo sapiens, Rattus norvegicus and Mus musculus). The two elements that were confirmed as true NR2F2 binding sites by chromatin immunoprecipitation are indicated with red lines. (C,D) Schematic scaled representation of the HEY1 (A) and HEY2 (B) loci highlighting the predicted NR2F2 binding elements (in green and red) and the sites (indicated by arrowheads) used to analyse binding of NR2F2 to these elements. qRT-PCR following chromatin immunoprecipitation with an anti-NR2F2 antibody revealed binding of NR2F2 to both genes in HUVECs (yellow) and LECs (blue) in site 1 containing the red NR2F2 element (lane 2, site 1) but not in site 2 (lane 2, site 2) Input is shown in lane 1, IgG-treated samples were used as negative control for precipitation (lane 3). Corresponding quantification (data expressed as fold enrichment \pm s.e.m. versus IgG) is shown on the right. Note the lower binding efficiency to the HEY2 promoter in LECs versus HUVECs. Chromatin immunoprecipitation with an anti-PROX1 antibody revealed binding of PROX1 to both genes at the same sites as NR2F2 in LECs (shaded blue; right). (E) Diagram showing luciferase (luc) assays in HUVECs revealing that mutating (Mut; black) the NR2F2 elements at site 1, shown in red in A-D, resulted in increased HEY1 and HEY2 promoter activity, respectively, compared with the corresponding wild-type (wt) control condition (white). Data are expressed as mean percentage activity \pm s.e.m. versus wild type $(N=3-5$ independent experiments; $* * P<0.01$ versus wild type). (F) qRT-PCR diagram revealing that expression of $H E Y 1 / 2$ is not affected by RBPJ siRNA treatment (black) in LECs [ $N=3$ independent experiments; data are expressed as mean percentage \pm s.e.m. versus nonsilencing siNS control (white)]. (G) qRT-PCR diagram revealing that expression of $H E Y 1 / 2$ is not affected by DAPT treatment (black) in LECs [ $N=3$ independent experiments; data are expressed as mean percentage \pm s.e.m. versus DMSO control (white)]

likely involved in basal Notch target gene expression in cultured LECs.

\section{NR2F2 forms homodimers in HUVECs but heterodimerises with PROX1 in LECs}

As differential DNA binding efficiency did not entirely explain NR2F2's divergent effect on HEY repression in HUVECs and LECs, we hypothesised that the inability of NR2F2 to repress $H E Y$ in LECs is related to its association with PROX1, as such an association was recently shown (Lee et al., 2009; Yamazaki et al., 2009) (Fig. 3A). Previous studies in non-ECs demonstrated that NR2F2 forms stable homodimers and that each monomer binds to one half-site of the NR2F2 binding element (Cooney et al., 1992; Pereira et al., 2000). Using a proximity ligation assay (PLA), we demonstrated that NR2F2 also associates with itself in HUVEC nuclei (Fig. 3B-D). In contrast, NR2F2 homodimers were nearly undetectable in LEC nuclei, but numerous NR2F2/PROX1 heterodimers were present there (Fig. 3C,F,G). NR2F2/PROX1 heterodimeric complex formation at the $H E Y$ promoters was further suggested by the observation that PROX1 binding occurred at the same sites as NR2F2 in LECs (Fig. 2C,D). Interestingly, NR2F2 homodimers were predominantly located in the cytoplasm rather than the nucleus, while only few NR2F2/ PROX1 heterodimers were found in the cytoplasm in LECs (Fig. 3C,F,G). Thus, the unique expression of PROX1 caused a switch from NR2F2 homo- to heterodimerisation in LEC nuclei.

\section{NR2F2 homo- and heterodimers differentially regulate gene expression in HUVECs and LECs}

We next tested whether the switch from NR2F2 homodimerisation in HUVECs to heterodimerisation with PROX1 in LECs was responsible for the inability of NR2F2 to repress $H E Y$ genes and its ability to activate LEC-specific genes. We therefore modified the NR2F2/PROX1 expression balance by siRNA knockdown or 

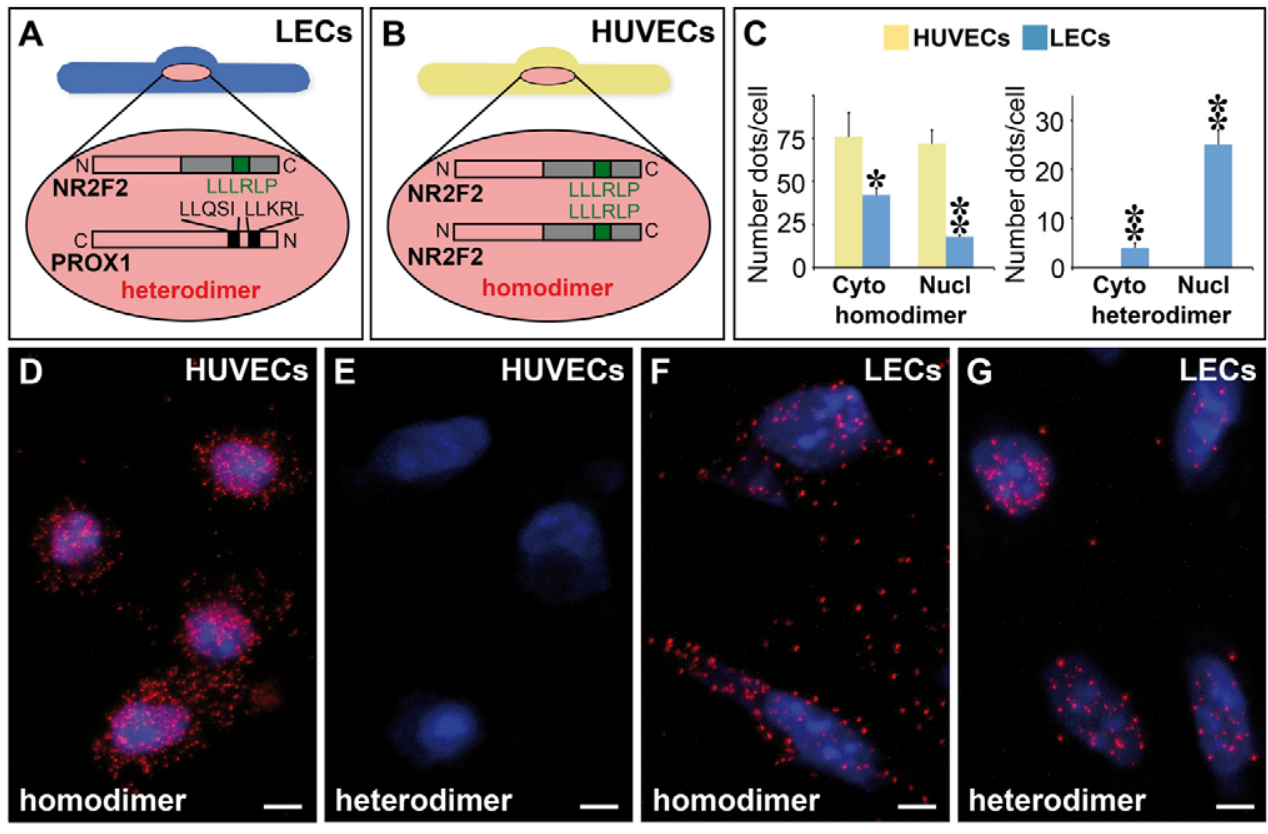

Fig. 3. NR2F2 forms homodimers in HUVECs and heterodimers with PROX1 in LECs. (A,B) Schematic diagram showing heterodimerisation between NR2F2 and PROX1 in LECs (A) and homodimer formation by NR2F2 in HUVECs (B). LLLRLP is the amino acid sequence in the ligand binding domain (grey box) at the C-terminus ('C') of NR2F2 that is important for dimerisation (Kruse et al., 2008). LRKLL and ISQLL are the amino acid sequences in the PROX1 N-terminus (N) implicated in heterodimerisation with nuclear receptors (Charest-Marcotte et al., 2010), supposedly including NR2F2 (Lee et al., 2009).

(C-G) Proximity ligation assay (PLA) revealing that NR2F2 forms homodimers (red dots in D) in the nucleus (Nucl in C; stained with Topro in blue in D-G) and cytoplasm (Cyto in C), but not NR2F2/PROX1 heterodimers in HUVECs (E; yellow in C). In LECs (blue in C), NR2F2 mainly forms NR2F2/PROX1

heterodimers (red dots in $\mathrm{G}$ ) in addition to a few homodimers (F). Note that in contrast with HUVECs, NR2F2 homodimers in LECs preferentially localise in the cytoplasm (F). Data in $\mathrm{C}$ are expressed as mean number \pm s.e.m. $\left({ }^{*} P<0.05,{ }^{*} P<0.01\right.$ versus HUVECs). Scale bars: $5 \mu \mathrm{m}$.

lentiviral overexpression of PROX1. PLA showed that our manipulations yielded the intended shifts in the homo-/ heterodimer balance (Fig. 4A-E,G-K). When PROX1 was overexpressed in HUVECs to promote heterodimerisation (and mimic the situation in LECs), expression of $H E Y 1 / 2$ was increased (Fig. 4F) in addition to several others of the 125 'type A' genes that are - like $H E Y 1 / 2$ - normally inhibited by NR2F2 in HUVECs but not in LECs (Fig. 5A,B). By contrast, PROX1 knockdown in LECs to favour NR2F2 homodimerisation (like in HUVECs) inhibited expression of a sampled subset of these genes (including HEY1 but not HEY2; Fig. 4L; Fig. 5A,C). Consistent with the ability of NR2F2 to activate gene expression when forming heterodimers with PROX1, overexpression of the latter in HUVECs activated the expression of a sampled subset of the 105 'type B' LEC marker genes induced by NR2F2 in LECs only (Fig. 5A,D). Conversely, PROX1 knockdown inhibited the expression of these genes (Fig. 5A,E).

\section{Direct interference with heterodimer formation further supports its major role in differential gene expression} In order to complement these homo-heterodimer shifts, we performed experiments to more directly interfere with NR2F2/ PROX1 heterodimer formation. Therefore, we mutated the nuclear receptor (NR) domains in the PROX1 N-terminus (Fig. 6A), previously suggested (Lee et al., 2009; Srinivasan et al., 2010) - but not shown - to be responsible for interaction with nuclear receptors, including NR2F2, while not affecting PROX1's inductive capacity for LEC gene expression (Srinivasan et al., 2010). While we confirmed that this PROX1 mutant retains its activity to induce LEC genes, including $P D P N$, FLT4 and REELIN (RELN; Fig. 6B), the NR mutations did not abrogate heterodimerisation with NR2F2 upon overexpression in HUVECs (Fig. 6C-E). Accordingly, these mutations did not impair PROX1's ability to boost $H E Y$ expression (Fig. 6B). Since the PROX1 NR mutant was not an appropriate tool to directly affect heterodimerisation, we used an alternative approach, i.e. simultaneous overexpression of PROX1 and knockdown of NR2F2 in HUVECs, the latter to disrupt NR2F2/PROX1 heterodimers resulting from PROX1 overexpression (Fig. 7, scheme on the left). In support of a major role for homoversus heterodimerisation in the differential regulation of gene expression by NR2F2, for five out of six sampled 'type B' genes, heterodimer disruption abolished the expression boost induced by PROX1 overexpression (Fig. 7, bar diagrams on the right).

\section{PROX1 DNA binding is required for inducing expression of an NR2F2-regulated LEC gene subset}

Finally, we explored whether the formed NR2F2/PROX1 heterodimers were either permissive for or rather actively inducing the expression of 'type A' genes, the latter through binding of PROX1 to DNA. To test this, we overexpressed a PROX1 DNA binding-deficient mutant in HUVECs, which did not interfere with heterodimerisation (Fig. 8A-D). Expression of a subset of 'type A' genes, including $H E Y 1$, was dependent on PROX1 DNA binding for its induction, while for others, including HEY2, PROX1 DNA binding was not required (Fig. 8E, left). Similarly, for 'type B' genes, the expression boost by NR2F2/PROX1 heterodimerisation may be additionally 

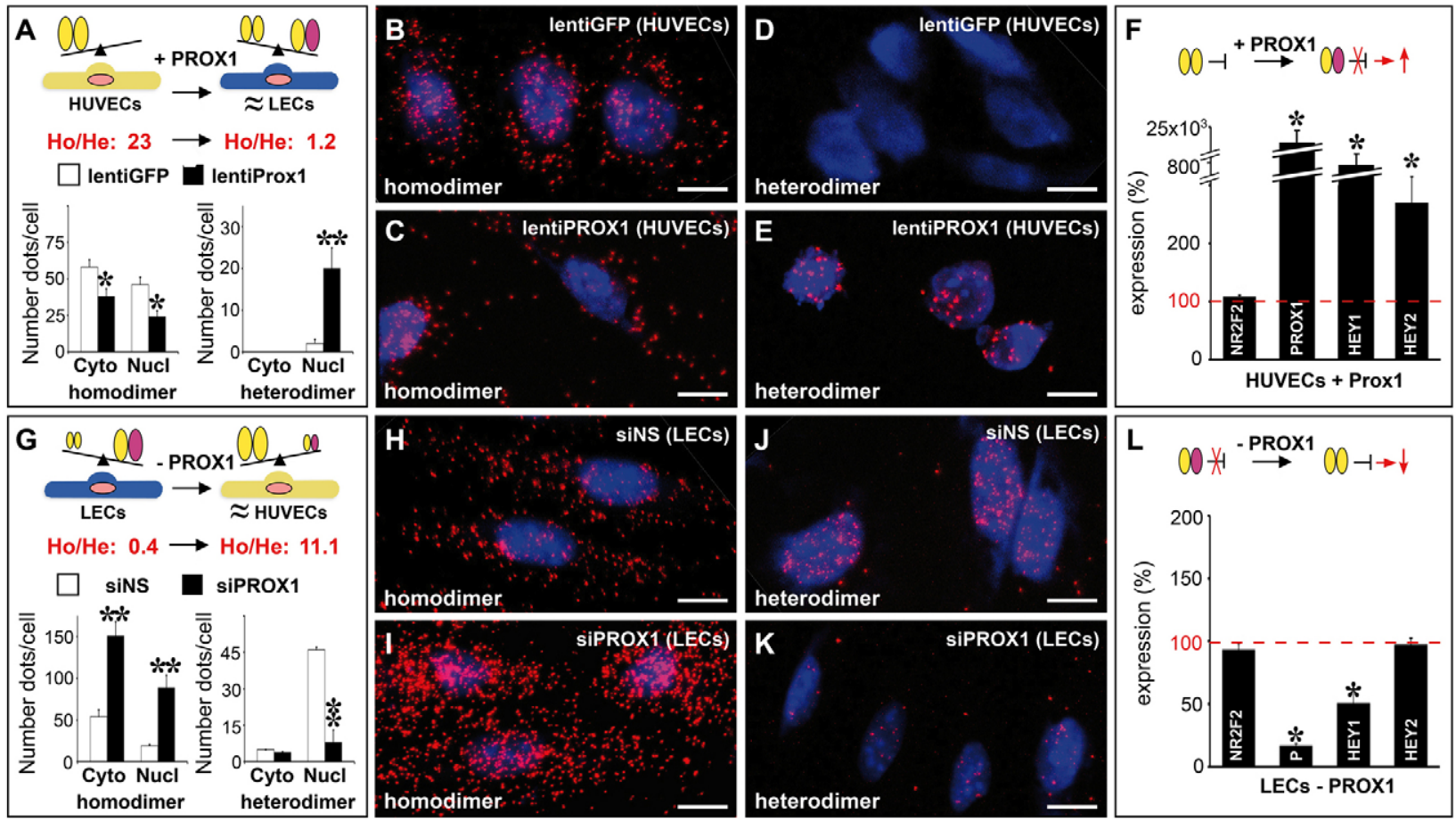

Fig. 4. NR2F2 homo- and heterodimerisation differentially regulate HEY expression in HUVECs and LECs. (A-F) PROX1 overexpression in HUVECs. Proximity ligation assays (PLA) reveal that this manipulation results in a reduction of NR2F2 homodimers (dots in B-E; schematically shown as yellow ellipses in $\mathrm{A}, \mathrm{F}$ ) in the nucleus (Nucl; stained with Topro in blue) and - to a lesser extent - in the cytoplasm (Cyto; B,C) and a gain of NR2F2/PROX1 heterodimers (D,E; schematically shown as yellow/magenta ellipses) in the nucleus of HUVECs, resembling the situation in LECs. The corresponding quantification is shown in A (data represent mean number \pm s.e.m.; ${ }^{*} P<0.05,{ }^{*} P<0.01$ versus lentiGFP). The nuclear homo/heterodimer ratio $(\mathrm{Ho} / \mathrm{He})$ is significantly reduced in PROX1-overexpressing HUVECs (A). As a result of this shift, the blocking effect of NR2F2 homodimers on HEY1/2 expression is released (F: data represent mean percentage \pm s.e.m. versus lentiGFP; $N=6$ independent experiments; $* P<0.05$ versus lentiGFP). (G-L) PROX1 knockdown in LECs. PLA reveals that this manipulation causes a gain of homodimers in the nucleus and the cytoplasm $(\mathrm{H}, \mathrm{I})$ and a loss of NR2F2/PROX1 heterodimers in the nucleus (J,K) of LECs, resembling the situation in HUVECs. The corresponding quantification is shown in G [data represent mean number \pm s.e.m.; ${ }^{* *} P<0.01$ versus non-silencing (siNS) siRNA]. The nuclear homo/heterodimer ratio $(\mathrm{Ho} / \mathrm{He})$ is significantly increased in PROX1-depleted LECs $(\mathrm{G})$. As a result of this shift, the transcriptional block on $H E Y 1$ - but not on $H E Y 2$ - expression is restored (L: data represent mean percentage \pm s.e.m. versus siNS; $N=4$ independent experiments; $* P<0.05$ versus siNS). For clarity, expected expression changes for each of the manipulations is schematically shown at the top of $\mathrm{F}$ and $\mathrm{L}$. Scale bars: $10 \mu \mathrm{m}$.

dependent on binding of PROX1 to regulatory sequences in their DNA. Indeed, in a set of six sampled 'type B' genes, four out of six were significantly dependent on PROX1 DNA binding (Fig. 8E, right). Thus, PROX1 DNA binding-dependency superimposed another level of regulation on NR2F2/PROX1 heterodimerisation for gene expression in LECs.

\section{Discussion}

AEC, VEC and LEC identities are in part genetically predetermined. NR2F2 orchestrates arterio-venous and venolymphatic decisions during development, a role that is evolutionary conserved across vertebrates (Aranguren et al., 2011; Lee et al., 2009; Lin et al., 2010; Srinivasan et al., 2010; You et al., 2005). Here, we demonstrated a differential effect of NR2F2 on gene and functional regulation in VECs and LECs. We propose a model whereby homo- versus heterodimerisation with PROX1 majorly determines this differential regulation. In VECs, NR2F2 homodimers blocked arterial differentiation by directly repressing $H E Y$ genes through binding to their promoters. In LECs, NR2F2/PROX1 heterodimers did not block HEY expression and induced a complement of LEC signature genes. $H E Y$ expression in LECs was at least partly independent of canonical Notch signalling. Furthermore, for some genes, expression in LECs was additionally dependent on PROX1 DNA binding.

Our first principal finding was that NR2F2, commonly expressed in VECs and LECs, drives strikingly different genetic and functional programs in these EC subtypes. Transcriptome analysis revealed that of the 449 genes significantly affected by NR2F2 knockdown in HUVECs and/or LECs, $88 \%$ of these were differently regulated by NR2F2 between HUVECs and LECs. Among the 'type A' genes specifically upregulated by NR2F2 knockdown - thus transcriptionally blocked by NR2F2 - in HUVECs were Notch target genes $H E Y 1 / 2$, consistent with the previously published hypothesis that NR2F2 blocks Notch signalling in VECs (You et al., 2005). While according to that hypothesis NR2F2 blocked Notch in VECs by acting at the level of VEGF co-receptor Nrp1 (You et al., 2005), our studies revealed an alternative mechanism of directly blocking Notch target genes, i.e. by binding of NR2F2 to DNA binding elements in their promoter. Chen et al. recently reported an additional transcriptional repressive NR2F2 DR3 binding element in the HEY2 promoter in close vicinity to the IR1 binding element identified in our screen, suggesting that NR2F2-mediated HEY2 repression in 


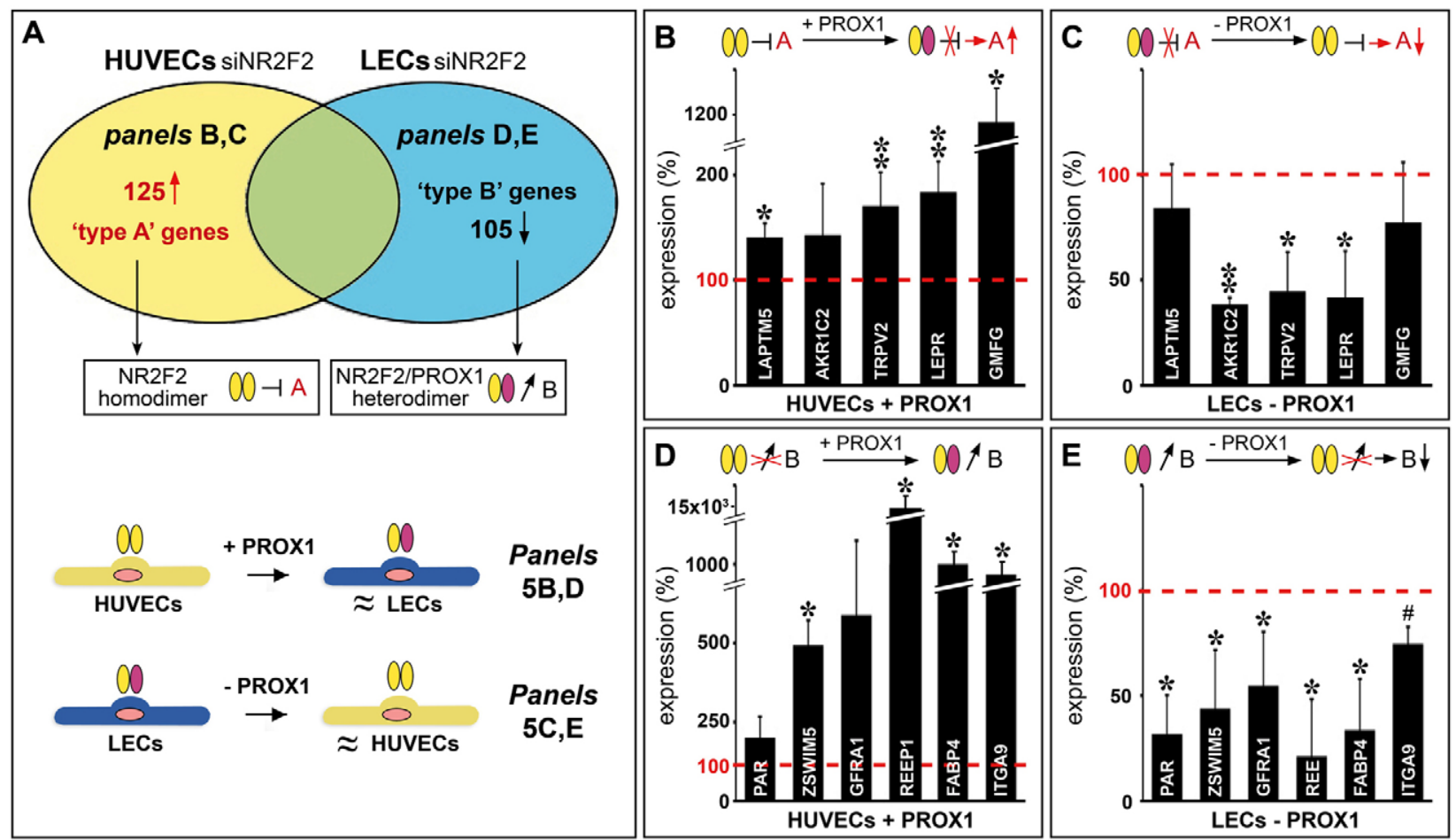

Fig. 5. NR2F2 homo- and heterodimerisation differentially regulate gene expression in HUVECs and LECs. (A) The upper diagram displays genes uniquely upregulated by NR2F2 siRNA knockdown in HUVECs ('type A' genes - in red - the transcription of which under baseline conditions is thus repressed by NR2F2 homodimers schematically shown as yellow ellipse pairs) or downregulated by NR2F2 siRNA knockdown in LECs ('type B' genes - in black - the transcription of which under baseline conditions is thus induced by NR2F2/PROX1 heterodimers schematically shown as yellow/magenta ellipse pairs). 'Type A' genes are further analysed by qRT-PCR in (B,C). 'Type B' genes are further analysed by qRT-PCR in (D,E). The lower diagrams show the two manipulations that were applied on HUVECs or LECs in order to shift between homo- and heterodimerisation. Results of PROX1 overexpression are shown in B and D, and those of PROX1 siRNA knockdown in C and E. (B-E) qRT-PCR diagrams revealing expressional changes upon PROX1 overexpression in HUVECs (B,D; a manipulation causing a shift to heterodimerisation similar to the situation in LECs) or PROX1 knockdown (C,E; a manipulation favouring homodimer formation, resembling the situation in HUVECs). For clarity, expected expression changes for each of these manipulations is schematically shown at the top of each panel. Data reveal that the majority of genes showed significant changes that are expected based on the homo/heterodimer shift hypothesis. Data expressed as percentages versus the corresponding baseline control (lentiGFP for PROX1 overexpression and non-silencing siRNA for PROX1 siRNA knockdown) in B-E represent the mean \pm s.e.m. of $4-6$ independent experiments $\left({ }^{*} P<0.05 ; * * P<0.01 ; \# P=0.053\right)$.

VECs requires interaction with multiple promoter binding sites (Chen et al., 2012). In contrast, even though NR2F2 also associated with the promoters of $H E Y 1 / 2$ in LECs, the latter were not transcriptionally repressed by NR2F2 in these cells. As a consequence, HEY1/2 expression was more than 10-fold higher in LECs compared with HUVECs. Furthermore, this also means that HEY1/2 and NR2F2 were co-expressed in LECs, unlike in AECs where NR2F2 expression in AECs is absent due to Notch activity involving HEY1/2 (Kang et al., 2010; Sörensen et al., 2009). We hypothesise that this may be explained by a dosage effect, whereby high HEY levels in AECs cause NR2F2 repression while lower basal levels in LECs do not. This dose dependency of HEY expression likely requires tight regulation.

How then is $H E Y$ expression differently regulated between AECs and LECs? The strikingly similar defects in AEC specification between mice deficient for Notch receptors/ ligands, Rbpjк and Hey1/2 indicate that in AECs, expression of Hey $1 / 2$ is the result of canonical Notch activation, requiring interaction of Rbpj $\kappa$ with the cleaved and nuclear translocated Notch receptor (Fischer et al., 2004; Krebs et al., 2004). We found for the first time that basal HEY1/2 expression in cultured LECs is established at least in part independent of Notch cleavage or RВРЈк. This is compatible with recent in vivo data failing to demonstrate canonical Notch activity in LEC (precursors) in zebrafish embryos (Geudens et al., 2010) and with the observation that lymphatic specification is independent of Rbpjк in mice (Srinivasan et al., 2010) and the not significantly affected $H E Y 1$ expression in LECs upon treatment with DAPT, a Notch cleavage blocker (Kang et al., 2010). NICD/ RBPJК independent regulation of $H E Y$ genes has been suggested to play a role in non-endothelial cell types (Sanalkumar et al., 2010) and in blood vascular ECs. Indeed, Hey2 expression is not altered in the endocardium of Rbpjк mutant mice (Timmerman et al., 2004). Moreover, VEGF-C binding to VEGFR3-expressing blood vascular ECs causes upregulation of $H E Y 1 / 2$ expression in the presence of Dll4-Fc - a Notch inhibitor preventing cleavage and nuclear translocation of the Notch receptor (Tammela et al., 2011). While a similar Notch cleavage-independent/VEGF-Cinduced cascade may occur in VEGFR3-expressing LECs, another recent study showed that blocking Notch signalling (using the same Notch inhibitor) affected LEC sprouting, suggesting that canonical Notch signalling may also be active in LECs under certain conditions (Zheng et al., 2011). Nevertheless, the potentially context-dependent role of 


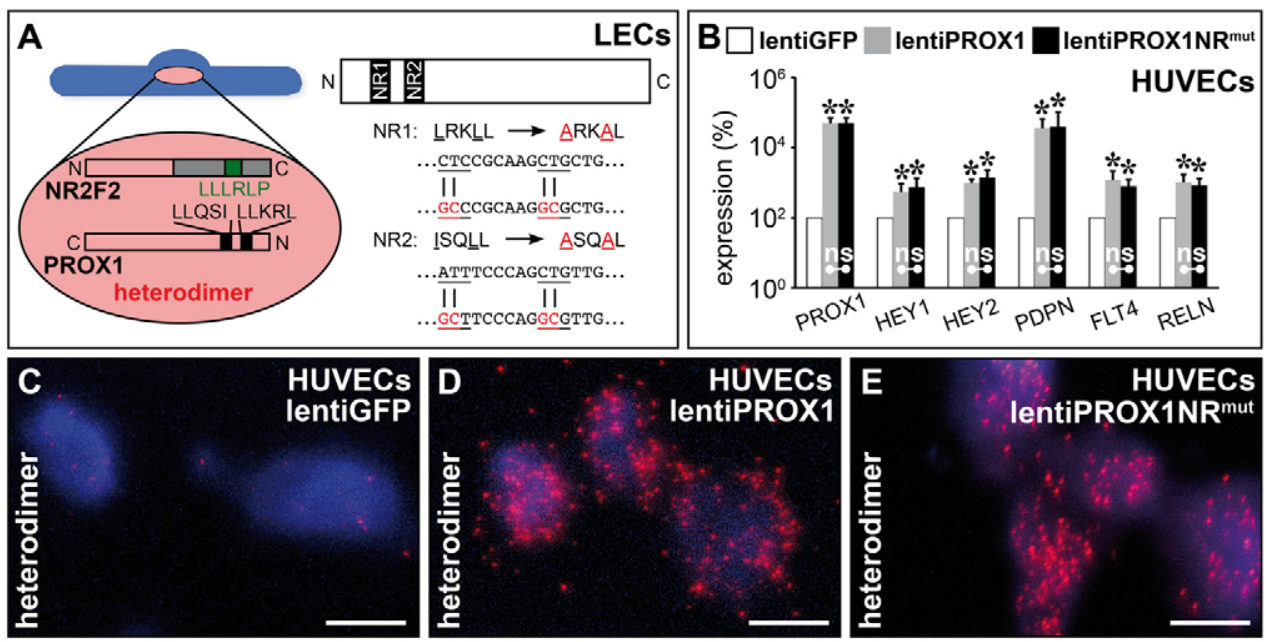

Fig. 6. NR1 and NR2 mutations in PROX1 do not affect NR2F2/PROX1 dimerisation. (A) Schematic representation of NR2F2/PROX1 heterodimerisation. According to the current hypothesis, two elements in the N-terminal region of PROX1 (NR1 corresponding to LRKLL and NR2 corresponding to ISQLL) are involved in binding to LLLRLP in the C-terminus of NR2F2. Mutations (in red font) were performed in both NR elements, replacing (iso)leucine with alanine, as described by Srinivasan et al. (Srinivasan et al., 2010). (B) Bar diagram representing the percentage expression \pm s.e.m. relative to lentiGFP in HUVECs treated with lentiGFP (white), lentiPROX1 (grey) or lentiPROX1NR ${ }^{\text {mut }}$ (black). Consistent with the comparable heterodimerisation capacity of the NR mutated and the wild-type PROX1, the NR mutations did not abrogate the heterodimerisation-induced increase in expression of HEY1/2 and LEC signature genes (podoplanin or PDPN, VEGFR3 or FLT4 and reelin or $R E L N)$. ${ }^{*} P<0.05$ versus lentiGFP; $N=3$ independent experiments; ns, not significant. (C-E) Proximity ligation assays revealing that the mutated PROX1 (PROX1NR ${ }^{\text {mut; }}$ E) was as efficient in forming heterodimers with NR2F2 as its wild type counterpart (D) upon overexpression in HUVECs. HUVECs transduced with lentiGFP (C) were used as a negative control. Scale bars: $10 \mu \mathrm{m}$.

canonical Notch signalling in LECs remains to be further elucidated (Geudens et al., 2010; Niessen et al., 2011; Srinivasan et al., 2010; Zheng et al., 2011). Recent studies demonstrated that BMP-9/Alk1 signalling induces HEY gene expression in ECs (Larrivée et al., 2012; Morikawa et al., 2011). Accordingly, our own (unpublished) findings suggest that HEY1/ 2 expression can be induced through BMP-9 signalling in LECs. Furthermore, Foxc1 and Foxc2 can activate HEY2 expression through direct binding of its promoter (Hayashi and Kume, 2008). Whether non-canonical HEY2 induction in LECs is Foxcmediated remains to be determined.

Another novel mechanistic finding of our study was that NR2F2 differentially regulates VEC and LEC function and gene expression by switching from homodimerisation in VECs to heterodimerisation with PROX1 in LECs. While NR2F2/PROX1 heterodimer formation has been previously demonstrated in LECs (Lee et al., 2009; Yamazaki et al., 2009), the consequences of this on the functional and transcriptional level have not been systematically analysed nor has there been a comparison with the role of NR2F2 (homodimers) in VECs. We used PLA in combination with overexpression or knockdown of PROX1 to demonstrate that the homo-heterodimer switch caused a striking difference in transcriptional regulation by NR2F2 in these EC subtypes. Unlike previous co-immunoprecipitation assays on cell lysates, PLA studies allowed us to evaluate the subcellular localisation of the interactions between individual NR2F2 and

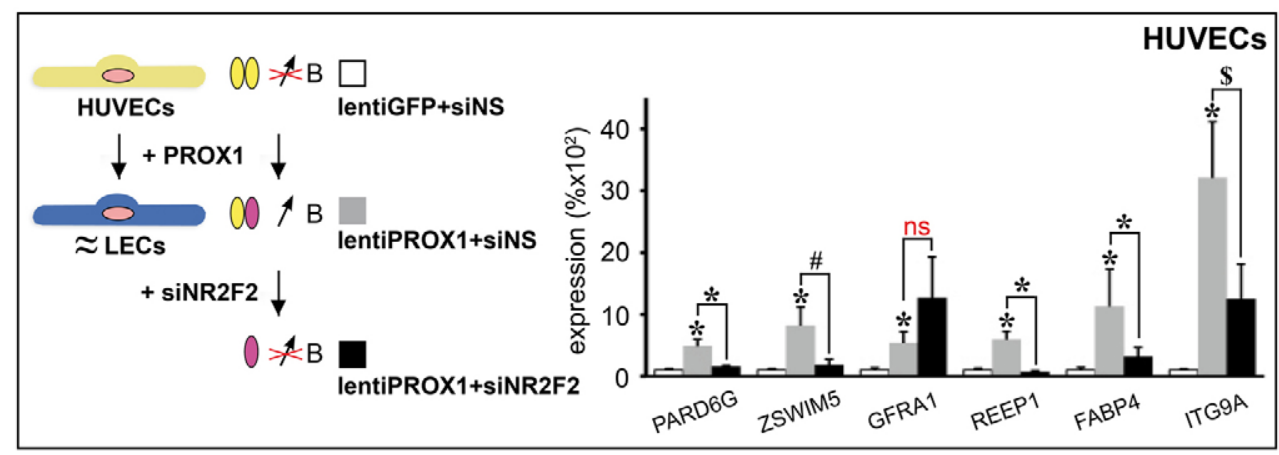

Fig. 7. Disruption of NR2F2/PROX1 heterodimers abrogates PROX1-induced induction of 'type B' genes. The left diagram schematically shows the expected expression changes for 'type B' genes upon PROX1 overexpression in HUVECs (to mimic the situation in LECs) and upon disruption of NR2F2/PROX1 heterodimers by NR2F2 siRNA knockdown. The qRT-PCR bar diagram on the right represents the percentage expression \pm s.e.m. relative to lentiGFP+siNS (nonsilencing siRNA) in HUVECs treated with lentiGFP+siNS (white), lentiPROX1+siNS (grey) or lentiPROX1+siNR2F2 (black), revealing that expression of the majority of randomly sampled 'type B' genes was dependent on heterodimerisation. $* P<0.05$ versus lentiGFP+siNS or lentiPROX1+siNS; $N=7$ independent experiments; ${ }^{\#} P=0.11$ versus lentiPROX1+siNS; ${ }^{\$} P=0.13$ versus lentiPROX1+siNS; ns, not significant. 

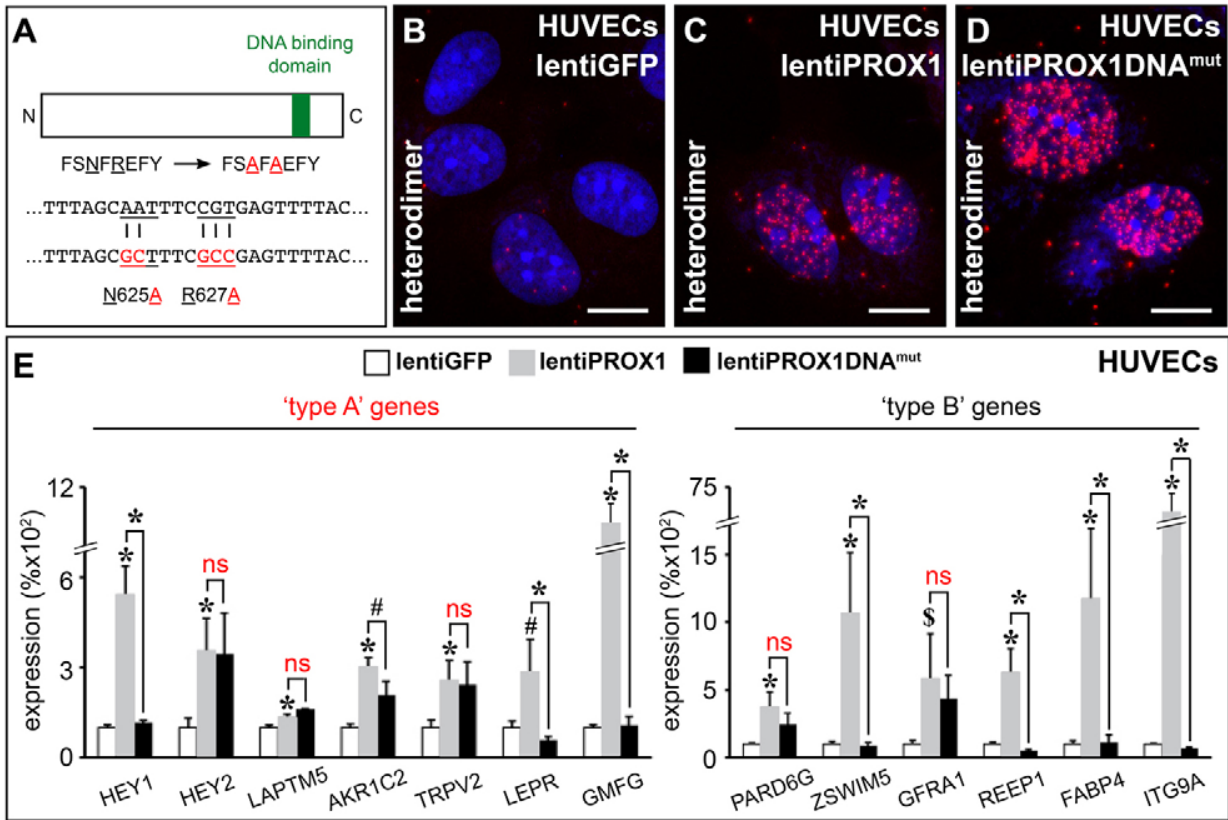

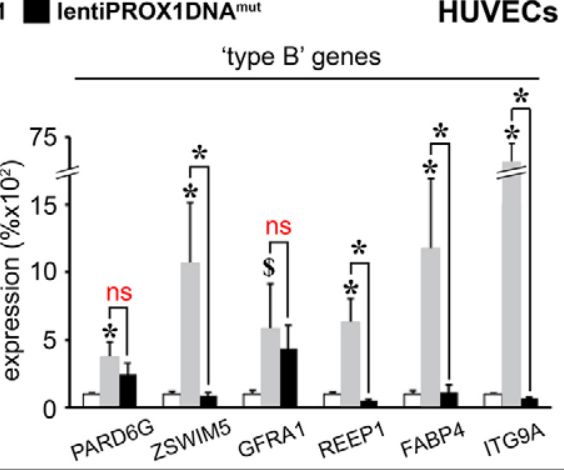

HUVECs
Fig. 8. PROX1 DNA-binding domain is required for expression of a subset of NR2F2regulated genes in LECs. (A) Schematic diagram showing the mutations (indicated by red font in the amino acid and corresponding DNA sequences) that were applied to disrupt PROX1 DNA binding. (B-D) Proximity ligation assays upon overexpression of the DNA bindingdeficient PROX1 mutant (lentiPROX1DNA ${ }^{\text {mut; }}$ D) or its wild-type equivalent (lentiPROX1; C) in HUVECs revealed that these mutations did not affect PROX1's ability to form heterodimers with NR2F2 (compared with the negative control lentiGFP in B). (E) qRT-PCR bar diagrams revealing that a subset of 'type A' genes (left) and a subset of 'type B' genes (right) was dependent on PROX1 DNA binding for their induction. Genes that were not dependent on PROX1 DNA binding are indicated by 'ns' in red font. Data are expressed as percentages versus the corresponding control and represent the mean \pm s.e.m. of six independent experiments $\left({ }^{*} P<0.05 ;{ }^{\#} P=0.06 ;{ }^{\$} P=0.1\right)$. Scale bars: $10 \mu \mathrm{m}$.
PROX1 molecules. Interestingly, NR2F2 homodimers were found both in HUVECs and LECs; however, in LECs these homodimers were less abundant and mainly resided in the cytoplasm. NR2F2/PROX1 heterodimers on the other hand were located almost exclusively in the LEC nuclei. The preferential presence of NR2F2 homodimers in the LEC cytoplasm may be explained by several mechanisms, including trapping by another protein, the existence of active shuttling between the nuclear and cytosolic compartments, or the fact that the observed homodimers are in transition to the nucleus where they switch to heterodimer formation.

A limitation of our study was that we could not confirm by mutations that disrupt the interaction between NR2F2 and PROX1 that the homo-heterodimerisation switch was responsible for the differential gene regulation in VECs versus LECs. This was due to the fact that the sequences in the PROX1 N-terminus implicated in heterodimerisation with nuclear receptors were not solely responsible for functional interaction of PROX1 with NR2F2 - as was demonstrated for its interaction with $E R R \alpha$, another nuclear receptor (Charest-Marcotte et al., 2010). Indeed, mutation of these sequences did not abolish NR2F2/PROX1 heterodimerisation nor did it affect $H E Y 1 / 2$ gene or LEC signature gene expression in established LECs. Notably, such mutations, however, did severely affect mouse lymphatic development (Srinivasan et al., 2010; Srinivasan and Oliver, 2011). Future studies to identify protein residues involved in functional dimerisation of PROX1 with nuclear receptors are warranted. Because of this limitation, we used an alternative strategy to directly interfere with NR2F2/PROX1 heterodimerisation by combining PROX1 overexpression with NR2F2 knockdown. This allowed us to demonstrate that homoversus heterodimerisation was a major contributor to the differential gene expression programs driven by NR2F2 in VECs versus LECs.

Finally, we observed that for a subset of 'type A' genes, PROX1's role was not merely to form a heterodimer with NR2F2 and thereby be permissive for their expression but rather to actively induce it by binding to regulatory DNA sequences somewhere in the gene. Thus, PROX1 DNA binding added another level of complexity on top of the heterodimerisation shift for gene expression regulation in LECs. Taking together the two sets of experiments where we either knocked down PROX1 in LECs or overexpressed a DNA binding-deficient mutant of PROX1 in HUVECs, we could distinguish at least three potential regulatory scenarios. For some genes, including $H E Y 1$, the NR2F2/PROX1 heterodimer was actively inducing their expression and this involved PROX1 DNA binding. For these genes, disrupting the heterodimer by PROX1 knockdown or interfering with the DNA binding capacity of PROX1 by overexpression of the mutant affected their expression. For others, like TRPV2, the shift to a heterodimer conformation was permissive and sufficient for their expression in LECs. For this gene category, the expression was affected by disruption of the heterodimer but not by interfering with PROX1 DNA binding. For yet another subset, including $H E Y 2$, the expression was neither affected by disruption of the heterodimer nor by interfering with PROX1 DNA binding. We postulate that for those genes the NR2F2/PROX1 heterodimer is permissive but their expression is additionally dependent on a trigger that does not involve PROX1. It will be interesting to identify this additional trigger for $H E Y 2$, which also should be independent of canonical Notch signalling, as its expression was not affected by inhibiting this pathway. Similarly to 'type A' genes, also for 'type B' genes the expression in LECs of a subset of them was dependent on PROX1-DNA interaction. The latter happened in the context of the NR2F2/PROX1 heterodimer, as PROX1 overexpression was no longer able to induce the expression of those genes when combined with NR2F2 knockdown. Thus, gene expression regulation by NR2F2 and PROX1 is a multi-level and complex process.

In summary, here we identified a novel mechanism by which a commonly expressed transcription factor, NR2F2, differentially regulated venous and lymphatic gene expression and function in VECs and LECs by homo- versus heterodimerisation with the 
LEC-specific transcription factor PROX1, respectively. Most intriguingly, unlike in VECs, NR2F2 did not block HEY1/2 expression in LECs and we demonstrated for the first time that their expression in LECs was at least in part independent of canonical Notch signalling. Furthermore, the expression of $H E Y$ genes as well as some others was dependent on an additional trigger in which PROX1 DNA binding was involved for a subset of these genes. Future studies will have to reveal the functional significance of these genes in LECs, most notably HEY genes, which were previously thought to be exclusively associated with arterial specification and function (Fischer et al., 2004; Gessler et al., 2002).

\section{Materials and Methods}

Cell derivation, culture, immunostaining, FACS and functional assays

HUVECs were obtained as described previously (Hendrickx et al., 2010). HUVEC isolation was performed at the Clinica Universidad de Navarra according to the institutional guidelines and after obtaining informed consent. Human lung LECs were purchased from Lonza (Merelbeke, Belgium), cultured in EBM-2 supplemented with EGM-2-MV bullet kit and subjected to a quality control [including FACS for Podoplanin, western blotting for PROX1 and immunofluorescence (IF) staining for NR2F2, PROX1, Podoplanin and integrin $9 \alpha$ ], revealing that they were a homogenous population expressing NR2F2 (data not shown). The IF staining and FACS procedures were described earlier (Aranguren et al., 2007) and primary antibodies used were: mouse anti-human NR2F2 (Perseus Proteomics PPH714700; 1:50; Tokyo, Japan), rabbit anti-human/mouse Prox1 (Angiobio 11-002; 1:100; Del Mar, CA, USA), rabbit anti-human/mouse Lyvel (Angiobio 11-034; 1:100), rabbit anti-human HEY1 (Santa Cruz (H120) SC28746; 1:20; Santa Cruz, CA, USA), rabbit anti-human HEY2 (Merck Millipore AB5716; 1:100; Overijse, Belgium), rabbit anti-human Podoplanin (Abcam ab 10274; 1:100; Cambridge, UK), and rabbit anti-human integrin $9 \alpha$ (Santa Cruz SC68864; 1:20). All IF images were recorded on a Zeiss Axiovert 40CFL microscope equipped with a Zeiss MRm camera and Axiovision 4.5 software (Carl Zeiss, Zaventem, Belgium). The scratch wound migration assay was performed as described (Hendrickx et al., 2010) in the presence of $100 \mathrm{ng} / \mathrm{ml}$ recombinant human VEGF-C (R\&D Systems, Abingdon, UK).

\section{Knockdown, lentiviral overexpression and canonical Notch blockade in ECs} siRNA knockdown was performed using Silencer ${ }^{\circledR}$ Select pre-designed siRNA from Applied Biosystems (Lennik, Belgium) for NR2F2 (siRNA ID\#: s62513 and s14021); negative control 1 (siRNA ID\#: am4636); PROXI (siRNA ID\#: s11229 and s11227) and $R B P J \kappa$ (siRNA ID\#: s7251 and s7253). Briefly, 5000 cells in $1.9 \mathrm{~cm}^{2}$ wells were plated on day 0 and transfected on day 1 with 5 pmol siRNA mixed with $0.5 \mu$ of Lipofectamine 2000 (Life Technologies, Gent, Belgium) in $100 \mu 1$ of OPTI-MEM (Life Technologies). The day after, transfection media was replaced and cells were maintained until day 3 or 6 , the latter requiring an additional siRNA transfection round at day 3 . In all cases negative control 1 siRNA-transfected cells were used as reference. Knockdown was verified by qRTPCR and western blot. Lentiviral constructs for PROX1 overexpression and GFP control virus were from GeneCopoeia (Rockville, USA) and lentiviruses were produced as described (Consiglio et al., 2004). Transduced cells were sorted (taking advantage of the IRES-GFP within the lentiviral constructs) into TRIzol ${ }^{\circledR}$ buffer 6 days later. For blocking Notch receptor cleavage, cells were treated with $60 \mu \mathrm{M}$ DAPT (Calbiochem, San Diego, USA) or DMSO (control).

\section{RNA isolation, qRT-PCR and western blotting}

Total RNA from (sorted) cell lysates was extracted using Trizol ${ }^{\circledR}$ reagent or RLT lysis buffer (Qiagen, Venlo, The Netherlands). mRNA was reverse transcribed using Superscript III Reverse Transcriptase (Life Technologies) and cDNA underwent 40 amplification rounds on an ABI PRISM 7700 cycler (Perkin Elmer Applied Biosystems, Foster City, CA) as described (Hendrickx et al., 2010) Primer sequences for qRT-PCR are listed in supplementary material Table S2. mRNA levels were normalised using GAPDH as housekeeping gene. For western blotting, samples were collected in RIPA buffer (Sigma-Aldrich, Bornem, Belgium) and protein concentration was measured by the BCA assay. $40 \mu \mathrm{g}$ of protein was used for blotting. Blot pictures were recorded with a Bio-Rad Chemidoc XRS+ molecular imager, equipped with Image Lab software (Bio-Rad Laboratories, Nazareth, Belgium). Antibodies used were: mouse anti-human NR2F2 (Perseus Proteomics PPH714700; 1:2000), rabbit anti-human/mouse PROX1 (Angiobio 11-002; 1:500), rabbit anti-human/mouse Lyve1 (Angiobio 11-034; 1:500) and mouse anti-human $\alpha$-tubulin (Sigma-Aldrich T6199; 1:1000).

\section{Microarray}

HUVECs or LECs were treated with siRNA against $N R 2 F 2$ or control siRNA $\left(N=4\right.$ for each condition) and total RNA was extracted in Trizol ${ }^{\circledR} 6$ days after transfection. RNA quality and concentration were determined using Pico chips on a Bioanalyser 2100 (Agilent Technologies, Diegem, Belgium). Samples were processed in collaboration with the Nucleomics Core Facility for gene expression analysis using the Affymetrix HG-U133 Plus 2.0 GeneChip Oligonucleotide Microarray (Affymetrix, Santa Clara, CA). Per sample, an amount of 100 ng of total RNA spiked with bacterial RNA transcript positive controls (Affymetrix) was amplified and labelled using the GeneChip 3' IVT express kit (Affymetrix). All steps were carried out according to the manufacturer's protocol (Affymetrix). A mixture of purified and fragmented biotinylated aRNA and hybridisation controls (Affymetrix) was hybridised on Affymetrix HG U133 Plus 2.0 arrays followed by staining and washing in a GeneChip ${ }^{\circledR}$ fluidics station 450 (Affymetrix) according to the manufacturer's procedures. To assess raw probe signal intensities, chips were scanned using a GeneChip ${ }^{\circledR}$ scanner 3000 (Affymetrix). Functional analysis of the array data was done using Ingenuity Pathway Analysis (Ingenuity Systems, Redwood City, CA).

\section{Chromatin immunoprecipitation}

ChIP was performed with the Transcription Factor Chromatin Immunoprecipitation Kit (Diagenode), according to the manufacturer's recommendations. Briefly, cells were harvested by trypsinisation, washed and fixed. Chromatin shearing was done in shearing buffer by sonication with a Bioruptor (Diagenode). The chromatin solution was clarified by centrifugation and an aliquot of $30 \mu \mathrm{l}$ of sheared chromatin was put aside for preparation of input sample. The remaining $60 \mu \mathrm{l}$ was immunoprecipitated using mouse monoclonal anti-NR2F2 antibody (Perseus Proteomics PPH714700, $8 \mu \mathrm{g}$ ), rabbit polyclonal anti-PROX1 antibody (Angiobio 11-002; $4 \mu \mathrm{g}$ ) or the corresponding isotype (Mouse IgG; Diagenode Kch819015; $8 \mu \mathrm{g}$; Rabbit IgG; Diagenode Kch903060; 4ug; Liège, Belgium). After reversing the cross-links, immunoprecipitated DNA fragments were extracted with phenol/chloroform/ isoamylalcohol, washed and dissolved in water. NHRscan (http://asp.ii.uib. no:8090/cgi-bin/NHR-scan/nhr_scan.cgi) and alibaba 2.1 software were used to screen for conserved NR2F2 binding elements. Primers for amplification of the immunoprecipitated DNA fragments were: HEYl, site 1: forward: 5'AGCGCAGGCCTGCCGCGGT3', reverse: 5'CCTCCCCGCACTCAGACTT3'; HEYl, site 2: forward: 5'ATGATTCCGTTTTTGCCTCA3', reverse: 5'CATTAAGCATAGTGGTTTGGAAAA3'; HEY2, site 1: forward: 5'ATCTTCACCTCCCGGACTCT3', reverse: 5'GTCAGCTCTGATGGTAGGAA3'; HEY2, site 2: forward: 5'TTGTTGTTTGCTTCTTTTGGAT3', reverse: 5'TCAAAAGCAGTTGGCACAAG3'. Gel pictures were recorded with a Vilber Lourmat gel documentation system (Vilber Lourmat, Marne-la-Vallée, France).

\section{Luciferase reporter assay and mutagenesis}

Human HEY1 and HEY2 promoters were cloned from BAC into the pGL3 plasmid (Promega, Leiden, The Netherlands). NR2F2 binding elements in site 1 in proximity to the translational start (Fig. 2A-D) were mutated using the QuikChange II XL Directed Mutagenesis Kit (Agilent Technologies, Diegem, Belgium). The DR4 element in the HEY1 promoter ( $5^{\prime}$ TGCCCTGCGCTGACCT3') was mutated to $5^{\prime} \mathrm{TaCaCTaCaCTaACtT3}$ ' and the IR1 element in the HEY2 promoter (5'GCGTCATTGATCT3') was mutated to 5'aCaTaATTaATCT3'. For the luciferase assay, 40,000 HUVECs were plated the day before transfection in a $1.9 \mathrm{~cm}^{2}$ well. Cells were co-transfected with Fugene HD (Roche, Vilvoorde, Belgium) using $450 \mathrm{ng}$ of pGL3-HEY1 or pGL3-HEY2 plasmid and $50 \mathrm{ng}$ TKRenilla. Twenty-four hours later, the luciferase/Renilla signal was measured using the Dual Luciferase Reporter Assay System (Promega) and a Microlumat LB96V Luminometer (Berthold Technologies, Vilvoorde, Belgium). Mutations in the PROX1 NR domains (Fig. 6A) and the PROX1 DNA-binding domain (Fig. 8A) were achieved as previously described (Shin et al., 2006; Srinivasan et al., 2010) using the above-mentioned mutagenesis kit.

\section{Proximity ligation assays}

In order to determine NR2F2 homodimerisation and/or heterodimerisation with PROX1, the Duolink II in situ Proximity Ligation Assay (Olink Bioscience, Uppsala, Sweden) was used. Briefly, to analyse NR2F2 homodimerisation $20 \mu \mathrm{g}$ of monoclonal NR2F2 antibody (Perseus Proteomics PHH714700) was labelled with Duolink Probe marker PLUS (positive oligonucleotide) and another $20 \mu \mathrm{g}$ with Duolink Probe marker MINUS (negative complementary oligonucleotide). To detect NR2F2/PROX1 heterodimers monoclonal mouse antibody against NR2F2 (Perseus Proteomics PHH714700) and polyclonal rabbit antibody against PROX1 (Angiobio 11-002) were used followed by Duolink II anti-mouse PLUS (positive oligonucleotide) and Duolink II anti-rabbit MINUS (negative complementary oligonucleotide) secondary antibodies. Corresponding IgGs were used as negative control (mouse IgG, Reliatech IgG-001, Wolfenbüttel, Germany; rabbit IgG, Sigma-Aldrich I5006). For both homodimers and heterodimers Duolink II Detection Reagent Orange was used to visualise protein interactions. The total number of signal dots was counted separately in the nucleus and the cytoplasm on confocal images recorded with a Zeiss LSM510 confocal microscope equipped with LSM510 software (Carl Zeiss)

\section{Statistical analysis}

Data, expressed as mean \pm s.e.m. comparing two groups, were analysed by Student's $t$-test. SPSS software was used for statistical analyses and differences 
were considered significant when $P<0.05$. Statistical analysis of the microarray data was performed in the $\mathrm{R}$ programming environment, in conjunction with the packages developed within the Bioconductor project (http://www.bioconductor.org). The analysis was based on the RMA expression levels of the probe sets that had at least once a present MAS 5.0 detection call. Differential expression was assessed via the moderated t-statistic, taking into account that the data was paired. To control the false discovery rate, multiple testing correction was performed and probes with a corrected $P$-value below 0.05 and $\mid \log 2$ siCOUP $-\log 2 \mathrm{NS} \mid>1$ were selected.

\section{Acknowledgements}

We thank P. Vandervoort, T. Vervoort, B. Hoekman, T. Koninckx for technical assistance. Microarray analyses were performed by the Nucleomics Core Facility (http://www.nucleomics.be).

\section{Author contributions}

X.L.A., M.B., G.C., C.W. and I.V. designed and performed experiments, analysed and interpreted data; A.L.N. and C.W. provided vital technical support and reagents; C.V. and M.G. assisted in writing the manuscript; A.L. designed experiments, interpreted data and wrote the manuscript.

\section{Funding}

This work was supported by the European Commission [grant numbers FP7-StG-IMAGINED 203291 to A.L., STREP-FP6-StrokeMAP to A.L. and C.M.V.]; the Fund for Scientific Research (FWO) [research grant numbers FWO 1.5.219.08N and G.0B09.13N to A.L., two post-doctoral fellowships to A.L. and X.L.A., and a pre-doctoral fellowship to I.V.]; the Lymphatic Research Foundation (LRF) [post-doctoral grant to X.L.A.]; the Agentschap voor Innovatie door Wetenschap en Technologie (IWT) [pre-doctoral fellowship to M.B.]; the KU Leuven Center of Excellence [grant number EF/05/013 to A.L.]; the KU Leuven [Program Financing grant number PF/10/014 to A.L.]; the KU Leuven Geconcerteerde Onderzoeksacties (GOA) [grant number GOA11/012]; Inter-University Attraction Poles (IUAP) [grant number P7/07-DevRepair]; and the Deutsche Forschungsgemeinschaft [Sonderforschungsbereich 688, TP A16 grant to M.G.].

\section{Supplementary material available online at}

http://jcs.biologists.org/lookup/suppl/doi:10.1242/jcs.116293/-/DC1

\section{References}

Aird, W. C. (2007a). Phenotypic heterogeneity of the endothelium: I. Structure, function, and mechanisms. Circ. Res. 100, 158-173.

Aird, W. C. (2007b). Phenotypic heterogeneity of the endothelium: II. Representative vascular beds. Circ. Res. 100, 174-190.

Aranguren, X. L., Luttun, A., Clavel, C., Moreno, C., Abizanda, G., Barajas, M. A., Pelacho, B., Uriz, M., Araña, M., Echavarri, A. et al. (2007). In vitro and in vivo arterial differentiation of human multipotent adult progenitor cells. Blood 109, 26342642

Aranguren, X. L., Beerens, M., Vandevelde, W., Dewerchin, M., Carmeliet, P. and Luttun, A. (2011). Transcription factor COUP-TFII is indispensable for venous and lymphatic development in zebrafish and Xenopus laevis. Biochem. Biophys. Res. Commun. 410, 121-126.

Charest-Marcotte, A., Dufour, C. R., Wilson, B. J., Tremblay, A. M., Eichner, L. J., Arlow, D. H., Mootha, V. K. and Giguère, V. (2010). The homeobox protein Prox 1 is a negative modulator of ERRalpha/PGC-1alpha bioenergetic functions. Genes Dev. 24, 537-542.

Chen, X., Qin, J., Cheng, C. M., Tsai, M. J. and Tsai, S. Y. (2012). COUP-TFII is a major regulator of cell cycle and Notch signaling pathways. Mol. Endocrinol. 26, 1268-1277.

Consiglio, A., Gritti, A., Dolcetta, D., Follenzi, A., Bordignon, C., Gage, F. H., Vescovi, A. L. and Naldini, L. (2004). Robust in vivo gene transfer into adult mammalian neural stem cells by lentiviral vectors. Proc. Natl. Acad. Sci. USA 101, 14835-14840.

Cooney, A. J., Tsai, S. Y., O'Malley, B. W. and Tsai, M. J. (1992). Chicken ovalbumin upstream promoter transcription factor (COUP-TF) dimers bind to different GGTCA response elements, allowing COUP-TF to repress hormonal induction of the vitamin D3, thyroid hormone, and retinoic acid receptors. Mol. Cell. Biol. 12, 4153-4163.

Corada, M., Nyquist, D., Orsenigo, F., Caprini, A., Giampietro, C., Taketo, M. M., Iruela-Arispe, M. L., Adams, R. H. and Dejana, E. (2010). The Wnt/beta-catenin pathway modulates vascular remodeling and specification by upregulating D114/Notch signaling. Dev. Cell 18, 938-949.
Fischer, A., Schumacher, N., Maier, M., Sendtner, M. and Gessler, M. (2004). The Notch target genes Hey1 and Hey2 are required for embryonic vascular development. Genes Dev. 18, 901-911.

Gessler, M., Knobeloch, K. P., Helisch, A., Amann, K., Schumacher, N., Rohde, E., Fischer, A. and Leimeister, C. (2002). Mouse gridlock: no aortic coarctation or deficiency, but fatal cardiac defects in Hey2 -/- mice. Curr. Biol. 12, 1601-1604.

Geudens, I., Herpers, R., Hermans, K., Segura, I., Ruiz de Almodovar, C., Bussmann, J., De Smet, F., Vandevelde, W., Hogan, B. M., Siekmann, A. et al. (2010). Role of delta-like-4/Notch in the formation and wiring of the lymphatic network in zebrafish. Arterioscler. Thromb. Vasc. Biol. 30, 1695-1702.

Hayashi, H. and Kume, T. (2008). Foxc transcription factors directly regulate Dll4 and Hey2 expression by interacting with the VEGF-Notch signaling pathways in endothelial cells. PLOS ONE 3, e2401.

Hendrickx, B., Verdonck, K., Van den Berge, S., Dickens, S., Eriksson, E., Vranckx, J. J. and Luttun, A. (2010). Integration of blood outgrowth endothelial cells in dermal fibroblast sheets promotes full thickness wound healing. Stem Cells 28, 1165-1177.

Kang, J., Yoo, J., Lee, S., Tang, W., Aguilar, B., Ramu, S., Choi, I., Otu, H. H., Shin, J. W., Dotto, G. P. et al. (2010). An exquisite cross-control mechanism among endothelial cell fate regulators directs the plasticity and heterogeneity of lymphatic endothelial cells. Blood 116, 140-150.

Krebs, L. T., Shutter, J. R., Tanigaki, K., Honjo, T., Stark, K. L. and Gridley, T. (2004). Haploinsufficient lethality and formation of arteriovenous malformations in Notch pathway mutants. Genes Dev. 18, 2469-2473.

Kruse, S. W., Suino-Powell, K., Zhou, X. E., Kretschman, J. E., Reynolds, R., Vonrhein, C., Xu, Y., Wang, L., Tsai, S. Y., Tsai, M. J. et al. (2008). Identification of COUP-TFII orphan nuclear receptor as a retinoic acid-activated receptor. PLoS Biol. 6, e227.

Larrivée, B., Prahst, C., Gordon, E., del Toro, R., Mathivet, T., Duarte, A., Simons, M. and Eichmann, A. (2012). ALK1 signaling inhibits angiogenesis by cooperating with the Notch pathway. Dev. Cell 22, 489-500.

Lee, S., Kang, J., Yoo, J., Ganesan, S. K., Cook, S. C., Aguilar, B., Ramu, S., Lee, J. and Hong, Y. K. (2009). Prox1 physically and functionally interacts with COUPTFII to specify lymphatic endothelial cell fate. Blood 113, 1856-1859.

Li, L., Xie, X., Qin, J., Jeha, G. S., Saha, P. K., Yan, J., Haueter, C. M., Chan, L., Tsai, S. Y. and Tsai, M. J. (2009). The nuclear orphan receptor COUP-TFII plays an essential role in adipogenesis, glucose homeostasis, and energy metabolism. Cell Metab. 9, 77-87.

Lin, F. J., Chen, X., Qin, J., Hong, Y. K., Tsai, M. J. and Tsai, S. Y. (2010). Direct transcriptional regulation of neuropilin-2 by COUP-TFII modulates multiple steps in murine lymphatic vessel development. J. Clin. Invest. 120, 1694-1707.

Morikawa, M., Koinuma, D., Tsutsumi, S., Vasilaki, E., Kanki, Y., Heldin, C.-H., Aburatani, H. and Miyazono, K. (2011). ChIP-seq reveals cell type-specific binding patterns of BMP-specific Smads and a novel binding motif. Nucleic Acids Res. 39, $8712-8727$

Niessen, K., Zhang, G., Ridgway, J. B., Chen, H., Kolumam, G., Siebel, C. W. and Yan, M. (2011). The Notch1-D114 signaling pathway regulates mouse postnatal lymphatic development. Blood 118, 1989-1997.

Oliver, G. and Srinivasan, R. S. (2010). Endothelial cell plasticity: how to become and remain a lymphatic endothelial cell. Development 137, 363-372

Pereira, F. A., Qiu, Y., Zhou, G., Tsai, M. J. and Tsai, S. Y. (1999). The orphan nuclear receptor COUP-TFII is required for angiogenesis and heart development. Genes Dev. 13, 1037-1049.

Pereira, F. A., Tsai, M. J. and Tsai, S. Y. (2000). COUP-TF orphan nuclear receptors in development and differentiation. Cell. Mol. Life Sci. 57, 1388-1398.

Sanalkumar, R., Dhanesh, S. B. and James, J. (2010). Non-canonical activation of Notch signaling/target genes in vertebrates. Cell. Mol. Life Sci. 67, 2957-2968.

Shawber, C. J. and Kitajewski, J. (2008). Arterial regulators taken up by lymphatics. Lymphat. Res. Biol. 6, 139-143.

Shawber, C. J., Funahashi, Y., Francisco, E., Vorontchikhina, M., Kitamura, Y., Stowell, S. A., Borisenko, V., Feirt, N., Podgrabinska, S., Shiraishi, K. et al. (2007). Notch alters VEGF responsiveness in human and murine endothelial cells by direct regulation of VEGFR-3 expression. J. Clin. Invest. 117, 3369-3382.

Shin, J. W., Min, M., Larrieu-Lahargue, F., Canron, X., Kunstfeld, R., Nguyen, L., Henderson, J. E., Bikfalvi, A., Detmar, M. and Hong, Y. K. (2006). Prox1 promotes lineage-specific expression of fibroblast growth factor (FGF) receptor-3 in lymphatic endothelium: a role for FGF signaling in lymphangiogenesis. Mol. Biol. Cell 17, 576-584

Sörensen, I., Adams, R. H. and Gossler, A. (2009). DLL1-mediated Notch activation regulates endothelial identity in mouse fetal arteries. Blood 113, 5680-5688.

Srinivasan, R. S. and Oliver, G. (2011). Prox1 dosage controls the number of lymphatic endothelial cell progenitors and the formation of the lymphovenous valves. Genes Dev. 25, 2187-2197.

Srinivasan, R. S., Dillard, M. E., Lagutin, O. V., Lin, F. J., Tsai, S., Tsai, M. J., Samokhvalov, I. M. and Oliver, G. (2007). Lineage tracing demonstrates the venous origin of the mammalian lymphatic vasculature. Genes Dev. 21, 2422-2432.

Srinivasan, R. S., Geng, X., Yang, Y., Wang, Y., Mukatira, S., Studer, M., Porto, M. P., Lagutin, O. and Oliver, G. (2010). The nuclear hormone receptor Coup-TFII is required for the initiation and early maintenance of Prox 1 expression in lymphatic endothelial cells. Genes Dev. 24, 696-707.

Swift, M. R. and Weinstein, B. M. (2009). Arterial-venous specification during development. Circ. Res. 104, 576-588.

Tammela, T., Zarkada, G., Nurmi, H., Jakobsson, L., Heinolainen, K., Tvorogov, D., Zheng, W., Franco, C. A., Murtomäki, A., Aranda, E. et al. (2011). VEGFR-3 
controls tip to stalk conversion at vessel fusion sites by reinforcing Notch signalling. Nat. Cell Biol. 13, 1202-1213.

Timmerman, L. A., Grego-Bessa, J., Raya, A., Bertrán, E., Pérez-Pomares, J. M. Díez, J., Aranda, S., Palomo, S., McCormick, F., Izpisúa-Belmonte, J. C. et al. (2004). Notch promotes epithelial-mesenchymal transition during cardiac development and oncogenic transformation. Genes Dev. 18, 99-115.

Tsai, S. Y. and Tsai, M. J. (1997). Chick ovalbumin upstream promoter-transcription factors (COUP-TFs): coming of age. Endocr. Rev. 18, 229-240.

Wang, H. U., Chen, Z. F. and Anderson, D. J. (1998). Molecular distinction an angiogenic interaction between embryonic arteries and veins revealed by ephrin-B2 and its receptor Eph-B4. Cell 93, 741-753.
Yamazaki, T., Yoshimatsu, Y., Morishita, Y., Miyazono, K. and Watabe, T. (2009). COUP-TFII regulates the functions of Prox 1 in lymphatic endothelial cells through direct interaction. Genes Cells $\mathbf{1 4}$ 425-434.

You, L. R., Lin, F. J., Lee, C. T., DeMayo, F. J., Tsai, M. J. and Tsai, S. Y. (2005). Suppression of Notch signalling by the COUP-TFII transcription factor regulates vein identity. Nature 435, 98-104

Zheng, W., Tammela, T., Yamamoto, M., Anisimov, A., Holopainen, T., Kaijalainen, S., Karpanen, T., Lehti, K., Ylä-Herttuala, S. and Alitalo, K. (2011). Notch restricts lymphatic vessel sprouting induced by vascular endothelial growth factor. Blood 118, 1154-1162. 